\title{
COPD research and treatment pioneer
}

\author{
Cite as: CMAJ 2017 October 23;189:E1320-1. doi: 10.1503/cmaj.109-5458
}

Posted on cmajnews.com on Oct. 2, 2017.

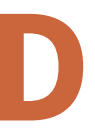

r. James Hogg has dedicated more than 40 years to researching, understanding and treating chronic obstructive pulmonary disease (COPD). His endeavors revolutionized the research and treatment of COPD and involved him in conducting scientific studies for the Royal Canadian Air Force. His accomplishments netted him the 2013 Canada Gairdner Wightman Award for scholarly contributions and he is a member of the Canadian Medical Hall of Fame.

Hogg is now professor emeritus at the Department of Pathology and Laboratory Medicine, University of British Columbia. At 81 , he continues to lead an active COPD research program at the Institute for Heart and Lung Health in Vancouver (originally called James Hogg Research Centre). Jonathon Chio recently interviewed Hogg about his multi-faceted career, which also includes roles as a health care policy maker and mentor.

What served as the catalyst for you to study lung disease and, in particular, COPD?

"When I was a second-year medical student in University of Manitoba, Dr. Joe Doupe - one of my physiology professors - gave us opportunities to participate in research projects. I was assigned to Dr. Reuben Cherniack, a famous pulmonary physiologist from University of Manitoba Medical School. This experience convinced me to pursue post-graduate research, such that in 1969, I completed a $\mathrm{PhD}$ in experimental medicine from McGill University. Currently, I am a card-carrying pathologist performing a mixture of diagnostic work on lung disease and research into lung structure and function."

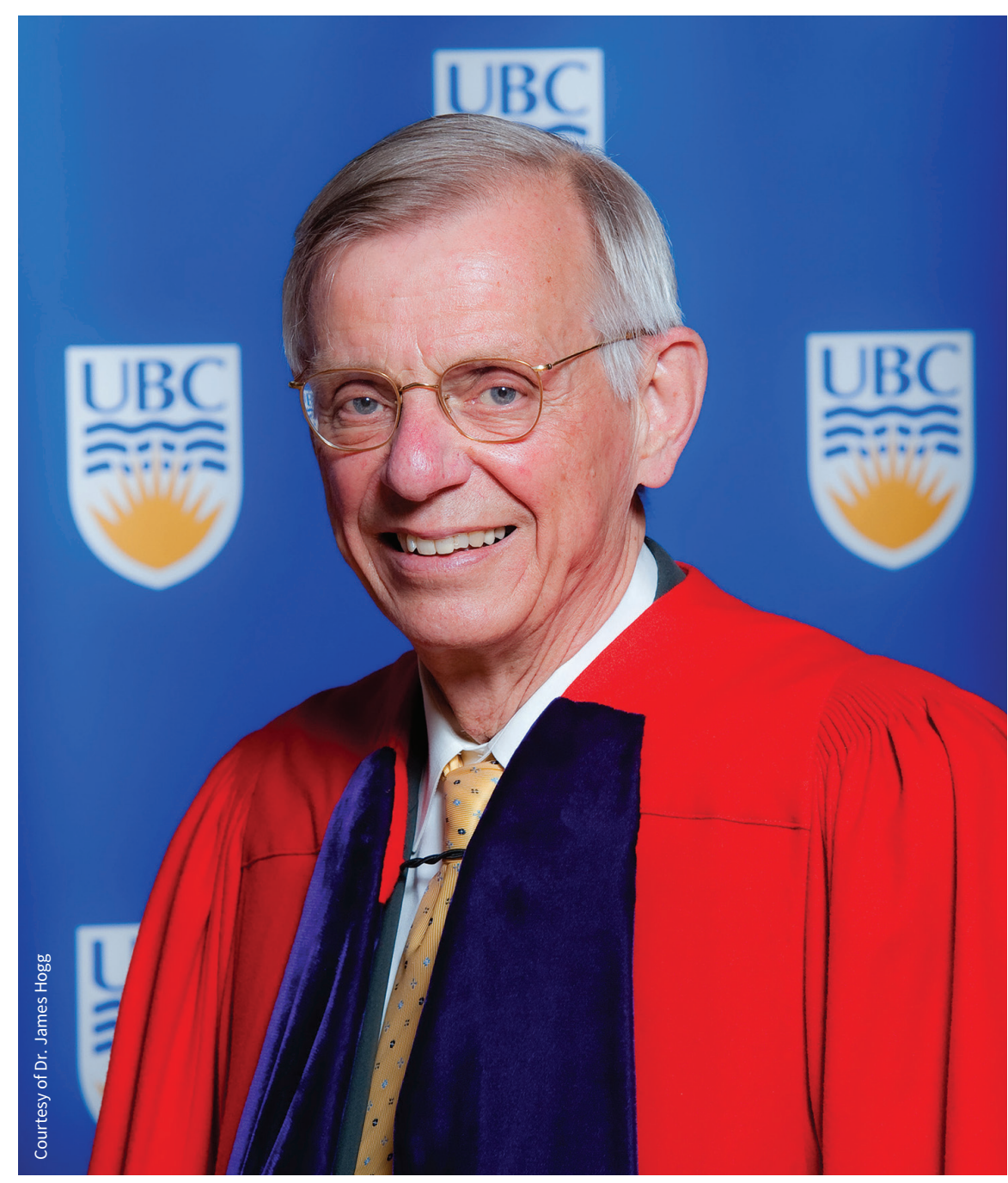

Dr. James Hogg spent 40 years working to improve the treatment of COPD.

Your first publication in 1968

presented the revolutionary idea of shifting the focus in COPD from large to small airways. What led to this

"eureka" moment?

"The moment came from my work with Dr. Joel Cooper. As a pathologist in the lung transplant program, I had access to human lungs. With Dr. Cooper, we developed a technique that allowed us to immediately inflate and freeze the lung after explanting from the patient. With these preserved lungs, we used threedimensional imaging strategies to make the exciting discovery that progressive destruction of terminal bronchioles is a major cause of COPD pathophysiology. Starting at very mild stages of disease, there is loss of about $20 \%$ of small airways. However, in severe patients, the 
loss becomes approximately $60 \%$ to $80 \%$. These observed changes led me to speculate that the increased small-airway resistance in patients with COPD is caused by loss of terminal bronchioles."

\section{Not many doctors have entered into} a centrifuge to experience and evaluate effects of g-forces on the lungs. What led you to pursue that area of research on subclinical coronary artery disease at the Royal Canadian Air Force?

"As a financially-stressed medical student, I was fortunate to have the Royal Canadian Air Force help cover my expenses. After graduation, I contributed back by completing three years of service, one of which was at the Institute of Aviation Medicine in Toronto. During the world wars, one of the biggest challenges faced by pilots operating high performance aircraft is the risk of fainting during the maneuvers. This was caused by the high gravitational forces that pool the blood in the legs and subsequently, deprive the brain of oxygen. Thus, the centrifuge was built in Toronto and allowed scientists - including myself to study what the pilots were experiencing and combine data from complementary murine models."

\section{What advice would you give to} health policy makers interested in implementing changes for the benefit of those affected by COPD (patient, family members, society)? "As COPD is a small-airway disease where terminal bronchioles are lost due to constant exposure to harmful particulates, one area of improvement is the antismoking policy. While it is well-established that smoking is harmful in adults, the impacts of smoking on young children are ill-elicited, especially effects from chronic exposure of fine particles on their small airways. Preliminary studies have shown that in children born into families with heavy smoking, they are significantly more probable to develop COPD later in life. Thus, as we learn more about COPD, we must change health care policies accordingly."
What advice would you give to aspiring researchers about achieving success in science without developing tunnel vision?

"While it is necessary to develop expertise in your field, one can avoid tunnel vision by participating in wide collaborations and being willing to learn. To be a successful scientist, it is important to be interested in your research and thrive as an independent individual. This passion will allow you to regard the persistent doubts and frustrations as a friend who is always there. Furthermore, as a scientist, you often have to solve problems on your own. Those who enjoy this aspect will likely be successful in the research they pursue."

Jonathon Chio, Toronto, Ont.

Editor's note: To commemorate the recent 30th anniversary of the Canadian Student Health Research Forum (CSHRF), four recipients of Gairdner awards gave public lectures highlighting their achievements. CSHRF participants were selected to interview the laureates. Jonathon Chio is a PhD student at the Institute of Medical Science at University of Toronto, Canada. His research focus is on devising novel immunomodulatory therapies to treat traumatic spinal cord injury. 